\title{
Review Article \\ Passive Microwave Component Design Using Inverse Scattering: Theory and Applications
}

\author{
Israel Arnedo, Iván Arregui, Magdalena Chudzik, Fernando Teberio, Aintzane Lujambio, \\ David Benito, Txema Lopetegi, and Miguel A. G. Laso
}

Electrical and Electronic Engineering Department, Public University of Navarre, Navarre 31006 Pamplona, Spain

Correspondence should be addressed to Israel Arnedo; israel.arnedo@unavarra.es

Received 15 March 2013; Accepted 22 May 2013

Academic Editor: Rocco Pierri

Copyright (c) 2013 Israel Arnedo et al. This is an open access article distributed under the Creative Commons Attribution License, which permits unrestricted use, distribution, and reproduction in any medium, provided the original work is properly cited.

\begin{abstract}
We briefly review different synthesis techniques for the design of passive microwave components with arbitrary frequency response, developed by our group during the last decade. We provide the theoretical foundations based on inverse scattering and coupledmode theory as well as several applications where the devices designed following those techniques have been successfully tested. The main characteristics of these synthesis methods are as follows. (a) They are direct, because it is not necessary to use lumpedelement circuit models; just the target frequency response is the starting point. (b) They are exact, as there is neither spurious bands nor degradation in the frequency response; hence, there is no bandwidth limitation. (c) They are flexible, because they are valid for any causal, stable, and passive transfer function; only inviolable physical principles must be guaranteed. A myriad of examples has been presented by our group in many different technologies for very relevant applications such as harmonic control of amplifiers, directional coupler with enhanced directivity and coupling, transmission-type dispersive delay lines for phase engineering, compact design of high-power spurious free low-pass waveguide filters for satellite payloads, pulse shapers for advanced UWB radar and communications and for novel breast cancer detection systems, transmission-type Nth-order differentiators for tunable pulse generation, and a robust filter design tool.
\end{abstract}

\section{Introduction}

The rigorous study of nonuniform microwave structures has been the starting point of the novel synthesis techniques of passive microwave components presented in this paper. The theoretical background rests on the knowledge of the coupled-mode theory, the field of periodic structures (PS), the electromagnetic band-gap (EBG) concepts, and the one-dimensional (1D) inverse scattering (IS) principles. The advantage of our proposals comes from the inherent properties of our synthesis approach and from the good performance achieved by structures with smooth (nonabrupt) profiles in terms of flexibility in the design [1], robustness in the implementation [2], spurious-multimode-excitation avoidance [3], and high-power handling capability [4].

Although the frequency response of PS [5] and EBG structures [6] feature a wide and deep rejected band (yielding to novel devices that have been found very promising applications, including the implementation of filters and resonators, improvement of the efficiency and radiation pattern of antennas, harmonic tuning in power amplifiers, oscillators and mixers, and suppression of spurious bands in filters [7]), they also exhibit characteristics that may be detrimental in other applications. Fortunately, some modifications on those structures [8] substantially ameliorate their behaviour. Windowing techniques [9] were reported to improve the out-of-band behaviour and chirping techniques [10] were used to enhance the bandwidth performance, while oversized structures were proposed to accomplish very high rejection levels over wide bandwidths [11].

Very useful to enrich the variety of target frequency responses attainable was the well-known Fourier transform relationship [12]. Unfortunately, this is only accurate enough for devices with very low reflectivity. Hence, using this relationship, the synthesis of a device with an arbitrary frequency response is only done in a first approximation. To surpass these difficulties, 1D-IS principles play a fundamental role. In this paper, an exact and analytical relationship between 
a generic frequency response and the physical dimensions of a device in the form of an infinite series is shown. Moreover, another exact and automatic alternative, valid when the target specifications can be expressed in terms of an arbitrary rational function, is proposed.

Four different synthesis approaches are sketched in Sections 2 and 3, while some devices synthesized following those methods are described in Section 4.

\section{Coupled-Mode Theory in Microwaves}

In order to deal with nonuniform microwave structures, it is essential to formulate an accurate coupled-mode theory suitable for microwave devices, where the crosssection method will be employed. The basic idea of this method is that the electromagnetic fields in an arbitrary nonuniform waveguide crosssection can be represented as a superposition of the different modes (including their forward and backward traveling waves) corresponding to a uniform auxiliary waveguide that has the same crosssection with identical distribution of $\varepsilon$ and $\mu[13,14]$. It is important to note that in most of the cases in the design of microwave devices, the problem can be greatly simplified by introducing several reasonable approximations that will lead to single-mode operation. Thus, the devices can be characterized by the following coupledmode system of equations:

$$
\begin{aligned}
& \frac{d a^{+}}{d z}=-j \cdot \beta \cdot a^{+}+K \cdot a^{-}, \\
& \frac{d a^{-}}{d z}=K \cdot a^{+}+j \cdot \beta \cdot a^{-},
\end{aligned}
$$

where

$$
\begin{gathered}
\widehat{\vec{E}}=a^{+} \cdot \vec{E}^{+}+a^{-} \cdot \vec{E}^{-}, \\
\widehat{\vec{H}}=a^{+} \cdot \vec{H}^{+}+a^{-} \cdot \vec{H}^{-}, \\
N^{+}=\iint_{S} \vec{E}^{+} \times \vec{H}^{+} \cdot d \vec{S}=-N^{-} \\
=-\iint_{S} \vec{E}^{-} \times \vec{H}^{-} \cdot d \vec{S}, \\
K=\frac{-1}{2 N^{+}} \iint_{S}\left(\vec{E}^{+} \times \frac{\partial \vec{H}^{-}}{\partial z}+\frac{\partial \vec{H}^{+}}{\partial z} \times \vec{E}^{-}\right) d \vec{S} \\
-\frac{1}{2 N^{+}} \cdot \frac{d N^{-}}{d z},
\end{gathered}
$$

being $\widehat{\vec{E}}, \widehat{\vec{H}}$ the total electric and magnetic field present in the structure; $\vec{E}^{+}, \vec{H}^{+}, \vec{E}^{-}$, and $\vec{H}^{-}$the vector mode patterns of the forward (+) and backward (-) traveling waves corresponding to the mode of operation in the auxiliary uniform waveguide associated with the crosssection of interest; $N^{+}, N^{-}$the normalizations taken for the fields of the mode; $\beta$ the phase constant of the mode; $K$ the coupling coefficient between the forward and backward traveling waves; $z$ the direction of propagation; and $a^{+}, a^{-}$, the complex amplitudes of the forward (+) and backward (-) traveling waves along the nonuniform waveguide.

Moreover, the physical dimensions of the device along the propagation direction can be easily deduced from the coupling coefficient once we choose a particular technology of implementation [13].

\section{Novel Synthesis Techniques}

The aim of all the synthesis methods presented here is to obtain the coupling coefficient of the device (or, equivalently, its physical dimensions once the technology is chosen) starting from some given frequency specifications. Depending on the strategy used, four kinds of methods arise.

3.1. Synthesis of Optimum Periodic Structures. If the structure is periodic along the propagation axis $z$ with period $\Lambda$, then the $K(z)$ produced by the perturbation is also periodic with the same period and, hence, it can be expanded in a Fourier series as follows:

$$
\begin{aligned}
K(z) & =\sum_{n=-\infty}^{n=\infty} K_{n} \cdot e^{j \cdot(2 \cdot \pi / \Lambda) \cdot n \cdot z} ; \\
K_{n} & =\frac{1}{\Lambda} \cdot \int_{\Lambda} K(z) \cdot e^{-j \cdot(2 \cdot \pi / \Lambda) \cdot n \cdot z} d z .
\end{aligned}
$$

For the system in (1a) and (1b), analytical expressions can be obtained for the central frequency, $f_{0, n}$, rejection level, $\left|S_{21}\right|_{\min , n}$, return loss level, $\left|S_{11}\right|_{\max , n}$, and bandwidth between zeros, $B W_{n}$, of the $n$th stopband produced by the two-port structure. For the case of planar structures (see [8]) we have the following:

$$
\begin{gathered}
f_{0, n}=\frac{c \cdot n}{2 \cdot \Lambda \cdot \sqrt{\varepsilon_{\mathrm{eff}}}}, \\
\left|S_{21}\right|_{\text {min }, n}=\operatorname{sech}\left(\left|K_{n}\right| \cdot L\right), \\
\left|S_{11}\right|_{\text {max }, n}=\tanh \left(\left|K_{n}\right| \cdot L\right), \\
B W_{n}=\frac{c \cdot\left|K_{n}\right|}{\pi \cdot \sqrt{\varepsilon_{\mathrm{eff}}}} \cdot \sqrt{1+\left(\frac{\pi}{\left|K_{n}\right| \cdot L}\right)^{2}},
\end{gathered}
$$

where $c$ is the speed of light in vacuum, $L$ is the device length, and $\varepsilon_{\text {eff }}$ is the mean value of $\varepsilon_{\text {eff }}(z)$ in a period, calculated as $\varepsilon_{\text {eff }}=\left(1 / \Lambda \cdot \int_{\Lambda} \sqrt{\varepsilon_{\text {eff }}(z)} \cdot d z\right)^{2}$.

These expressions can be used for the synthesis of microwave structures with ad hoc rejected bands, where we have independent control of their frequency, rejection level, and/or bandwidth. Of particular remarkable interest is the structure with spurious-free frequency response. Examining 
(5), the $n$th coefficient of the Fourier series, $K_{n}$, is univocally associated with the $n$th stopband and, therefore, to obtain an spurious-free EBG structure that features only the fundamental stopband, all the coefficients must be equal to zero except for $K_{ \pm 1}$. This results in a sinusoidal coupling coefficient following the generic expression:

$$
K(z)=A \cdot \cos \left(\frac{2 \cdot \pi}{\Lambda} \cdot z+\theta\right)
$$

where $A$ is the amplitude of the coupling coefficient and $\theta$ the phase that fixes its value at the origin.

3.2. Synthesis of Quasi-Periodic Structures: Windowed, Chirped, and Oversized Structures. In order to enrich the panel of frequency responses that can be satisfied by the synthesized structures, the purely periodic structure of sinusoidal coupling coefficient (6) can be windowed (by making the amplitude a smooth function of $z[9,15,16])$ or chirped (by making the period variable with $z[10,17])$. Finally, if the amplitude is high enough, a transversal resonance can be achieved, providing new degrees of freedom to the designer $[11,18]$.

3.3. General Microwave Synthesis Technique for an Arbitrary Frequency Response as a Solution of the Gel'fand-LevitanMarchenko 1D Inverse Scattering Problem. In order to synthesize a physical device with an arbitrary frequency response (just limited by causality, stability, and passivity), we start from the simplified system of coupled mode equations given in (1a) and (1b). This system can be rewritten as a ZakharovShabat system of quantum mechanics [19] for which an analytical solution can be found using the so-called Gel'fandLevitan-Marchenko coupled integral equations valid for $|z|>$ $\tau[19]:$

$$
\begin{gathered}
A_{1}(z, \tau)+\int_{-\tau}^{z} A_{2}^{*}(z, y) \cdot F(y+\tau) \cdot d y=0, \\
A_{2}(z, \tau)+F(z+\tau)+\int_{-\tau}^{z} A_{1}^{*}(z, y) \cdot F(y+\tau) \cdot d y=0,
\end{gathered}
$$

where $A_{1}(z, \tau)$ and $A_{2}(z, \tau)$ are auxiliary functions to be found and $F(\tau)$ is the inverse Fourier transform of the $S_{11}(\beta)$ parameter (starting specifications):

$$
F(\tau)=\frac{1}{2 \pi} \int_{-\infty}^{+\infty} S_{11}(\beta) \cdot e^{j \cdot \beta \cdot \tau} \cdot d \beta
$$

Thus, applying the Fourier Transform to (1a) and (1b) and using causality considerations, the coupling coefficient can be calculated as follows [19]:

$$
K(z)=2 \cdot A_{2}\left(z, z^{-}\right)
$$

Now, a series solution for $A_{2}(z, \tau)$ can be found by alternating approximations of $A_{1}(z, \tau)$ and $A_{2}(z, \tau)$ in (7a) and (7b) in the following way. We start by neglecting the integral term in (7b) and the first approximation for $A_{2}(z, \tau)$ is achieved. Introducing this result in $(7 \mathrm{a}), A_{1}(z, \tau)$ is updated and a new approximation of $A_{2}(z, \tau)$ is obtained. Iterating and taking finally into account that $F(\tau)$ will be real for a physical device, we arrive at the exact analytical series solution for the coupling coefficient given in (10), shown at the end of this subsection, where $x_{i}^{\prime}=x_{i}+x_{i-1}-2 z$ for $i>1$, that can be efficiently implemented with a computer, see [20]:

$$
\begin{aligned}
K(z)= & -2 F(2 z)-2 \int_{0}^{2 z} d x_{1} F\left(x_{1}\right) \int_{0}^{x_{1}} d x_{2}^{\prime} F\left(x_{2}^{\prime}\right) F\left(x_{2}^{\prime}-x_{1}+2 z\right)-2 \int_{0}^{2 z} d x_{1} F\left(x_{1}\right) \int_{0}^{x_{1}} d x_{2}^{\prime} F\left(x_{2}^{\prime}\right) \\
& \times \int_{0}^{x_{2}} d x_{3}^{\prime} F\left(x_{3}^{\prime}\right) \int_{0}^{x_{3}} d x_{4}^{\prime} F\left(x_{4}^{\prime}\right) F\left(x_{4}^{\prime}-x_{3}+2 z\right)-\cdots-2 \int_{0}^{2 z} d x_{1} F\left(x_{1}\right) \int_{0}^{x_{1}} d x_{2}^{\prime} F\left(x_{2}^{\prime}\right) \\
& \times \int_{0}^{x_{2}} \cdots \int_{0}^{x_{2 N-1}} d x_{2 N}^{\prime} F\left(x_{2 N}^{\prime}\right) F\left(x_{2 N}^{\prime}-x_{2 N-1}+2 z\right)-\cdots
\end{aligned}
$$

3.4. General Microwave Synthesis Technique for Rational Frequency Responses by Means of the 1D Inverse Scattering Problem in the Laplace Domain. In the case that the target frequency response can be expressed as a rational function, a fast synthesis method can be derived [21]. We will assume that $S_{11}(s)$ is a rational function that can be written as a quotient of polynomials as follows:

$$
S_{11}(s)=\frac{n(s)}{d(s)}=\frac{r_{0} \cdot \prod_{n=1}^{M}\left(s-c_{n}\right)}{\prod_{n=1}^{N}\left(s-p_{n}\right)}
$$

being $c_{n}$ the zeros and $p_{n}$ the poles of $S_{11}(s), M$ the number of zeros, and $N$ the number of poles, where it is satisfied that
$N \geq M+1\left(S_{11}(s=j \beta)\right.$ is a band limited function that tends to zero when frequency goes to infinity) and $\mathfrak{R} e\left\{p_{n}\right\}<0$ (all the poles must be in the left-half $s$ plane to be a stable system). Finally, $r_{0}$ is a constant that must be adjusted to satisfy $\mid S_{11}(s=$ $j \beta) \mid<1$ (passive system).

Hence, the $S_{21}$ parameter of the device arises univocally from the $S_{11}$ parameter for our case of interest of reciprocal and lossless two-port networks. Specifically, the magnitude can be calculated for each frequency (or equivalently $\beta$ ) as follows [22]:

$$
\left|S_{21}(s=j \beta)\right|^{2}=1-\left|S_{11}(s=j \beta)\right|^{2}
$$


yielding to the following:

$$
S_{21}(s)=\frac{\prod_{n=1}^{N}\left(s-k_{n}\right)}{\prod_{n=1}^{N}\left(s-p_{n}\right)}
$$

being $k_{n}$ the zeros and $p_{n}$ the poles of $S_{21}(s)$, where $\mathfrak{R} e\left\{k_{n}\right\}<$ 0 (all the zeros are in the left-half $s$ plane because $S_{21}(s)$ is a minimum-phase function) and $\mathfrak{R} e\left\{p_{n}\right\}<0$ (all the poles must be in the left-half $s$ plane to be a stable system).

Appling the Laplace transform to (9), the coupling coefficient can be calculated as follows [21]:

$$
K(z)=-2 \cdot \lim _{s \rightarrow-\infty}\left(e^{s \cdot z} \cdot s \cdot a_{2}(z, s)\right) .
$$

By inspecting (14), it can be seen that the coupling coefficient, $K(z)$, necessary to implement a given frequency response, can be calculated analytically by obtaining first an analytical solution for $a_{2}(z, s)$. To do it, we need to assume that the $N$ poles of $S_{11}(s)$ and $S_{21}(s), p_{1}, p_{2}, \ldots, p_{N}$, are distinct, that the $N$ zeros of $S_{21}(s), k_{1}, k_{2}, \ldots, k_{N}$, are distinct, and that the $M$ zeros of $S_{11}(s), c_{1}, c_{2}, \ldots, c_{M}$, are different from the conjugated poles with opposite sign, $-p_{1}^{*},-p_{2}^{*}, \ldots,-p_{N}^{*}$. Then, $a_{1}(z, s)$ and $a_{2}(z, s)$ can be expressed as a linear combination of entire functions [21] and, thus, the sought analytical closed-form expression for the coupling coefficient, $K(z)$, of the microwave structure that satisfies the target frequency response is obtained [21]:

$$
K(z)=2 \cdot \sum_{n=1}^{N}\left\{d_{2, n}(z) \cdot e^{k_{n} \cdot z}-d_{1, n}^{*}(z) \cdot e^{-k_{n}^{*} \cdot z} \cdot S_{11}\left(-k_{n}^{*}\right)\right\},
$$

where $d_{1, n}(z)$ and $d_{2, n}(z)$ are the solutions of the following linear system of $2 \cdot N$ equations [21]:

$$
\begin{aligned}
\sum_{n=1}^{N} & \left\{\frac{1}{S_{11}\left(k_{n}\right)} \cdot \frac{e^{-k_{n} \cdot z}}{p_{i}-k_{n}} \cdot\left[\begin{array}{l}
d_{1, n}(z) \\
d_{2, n}(z)
\end{array}\right]-\frac{e^{k_{n}^{*} \cdot z}}{p_{i}+k_{n}^{*}} \cdot\left[\begin{array}{l}
d_{2, n}^{*}(z) \\
d_{1, n}^{*}(z)
\end{array}\right]\right\} \\
& =\left[\begin{array}{l}
0 \\
1
\end{array}\right] .
\end{aligned}
$$

\section{Applications of the Synthesis Techniques}

Several devices have been designed following the abovementioned synthesis methods in different technologies. They are classified by their intended application.

4.1. Harmonic Control in Amplifier Applications. To check the efficiency of an amplifier, it is necessary to detect very tiny frequency spurious harmonics, usually masked by the fundamental carrier power. By using Section 3.1, a two-port spurious-free notch-filter can be designed [8], see Figures 1 and 2.

4.2. Directional Coupler with Enhanced Directivity and Coupling. Many microwave systems demand directional couplers with high directivity and coupling. A fully planar device

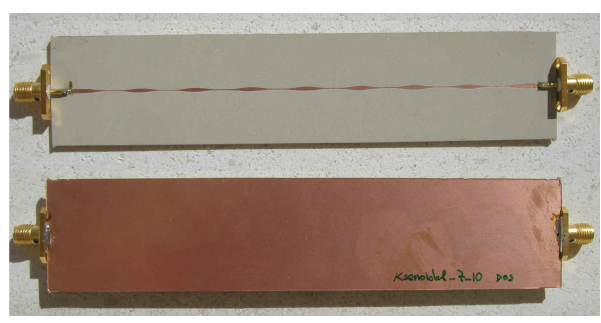

FIGURE 1: Photograph of the fabricated prototype in microstrip technology; top view of the metallic strip and bottom view of the unaltered ground plane.

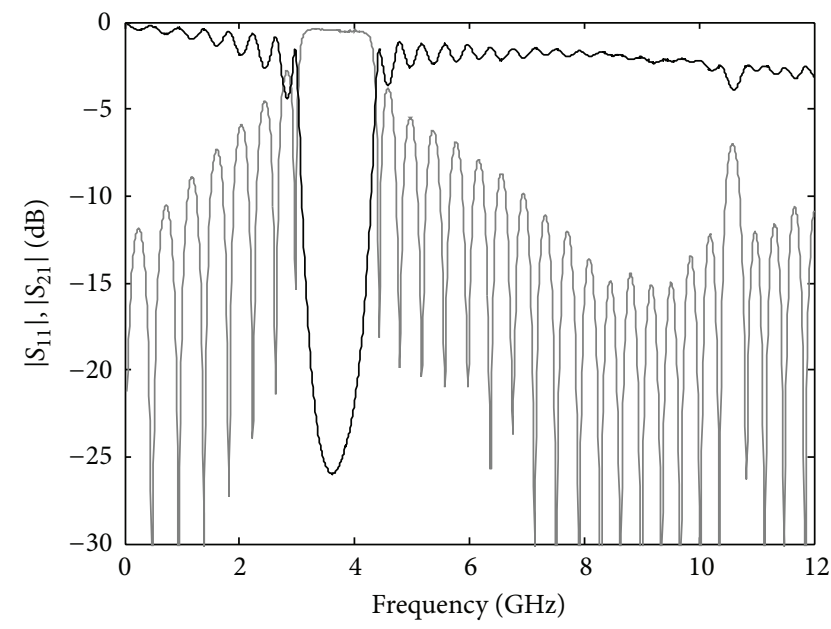

Figure 2: Measured $\left|S_{21}\right|$ (black line) and $\left|S_{11}\right|$ (grey line) parameter of the designed two-port EBG microstrip structure in Figure 1.

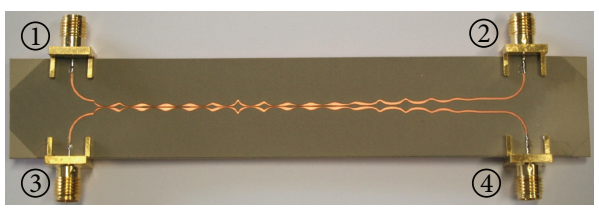

FIGURE 3: Photograph of the fabricated EBG microstrip directional coupler with high directivity and coupling. The ground plane is unaltered.

where coupling level and frequency can be obtained following the method Section 3.1 for a four-port structure [23], see Figures 3 and 4.

4.3. Transmission-Type Dispersive Delay Lines for PhaseEngineering Application. Phase engineering in microwaves has drawn very much attention in the last years [24]. It is devoted to the development of analog signal processing systems in the microwave frequency range with fundamental building blocks such as Dispersive Delay Lines of different kinds. Following the ideas of chirping and windowing in Section 3.2, a new transmission-type dispersive delay line can be proposed with closed formulas for the dispersion slope and bandwidth of operation [25], see Figures 5, 6, 7, and 8 . 


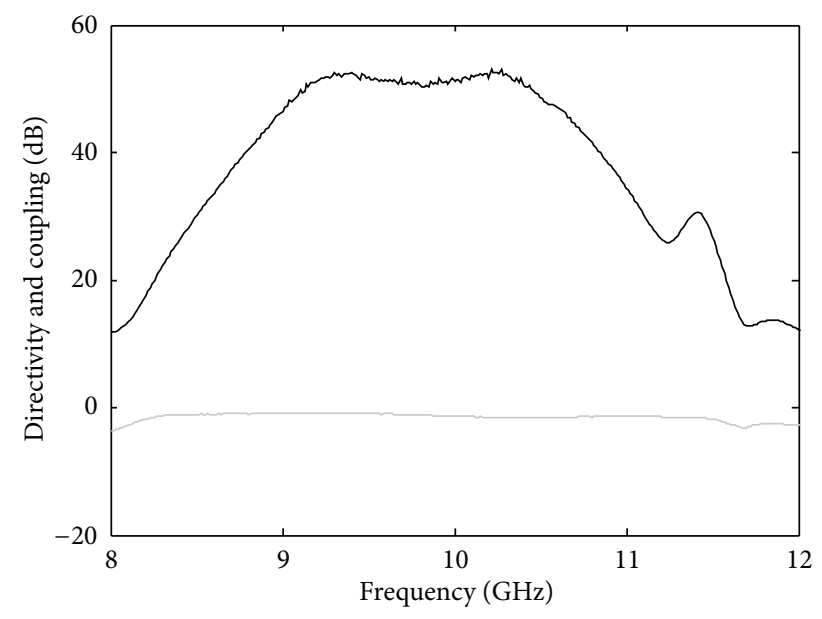

FIGURE 4: Measured directivity (black line) and coupling (grey line) of the designed EBG microstrip directional coupler in Figure 3.

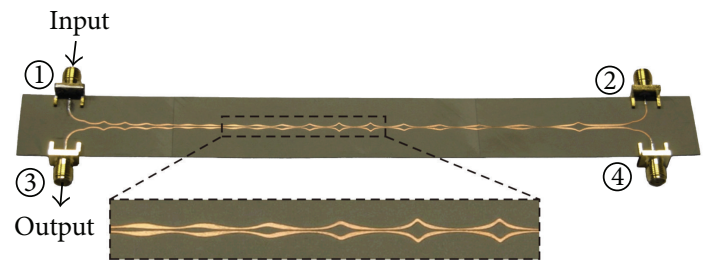

Figure 5: Photograph of the fabricated prototype of transmissiontype dispersive delay line, indicating the input and output ports. A zoom-in is included to show a detail of the device. The ground plane is unaltered.

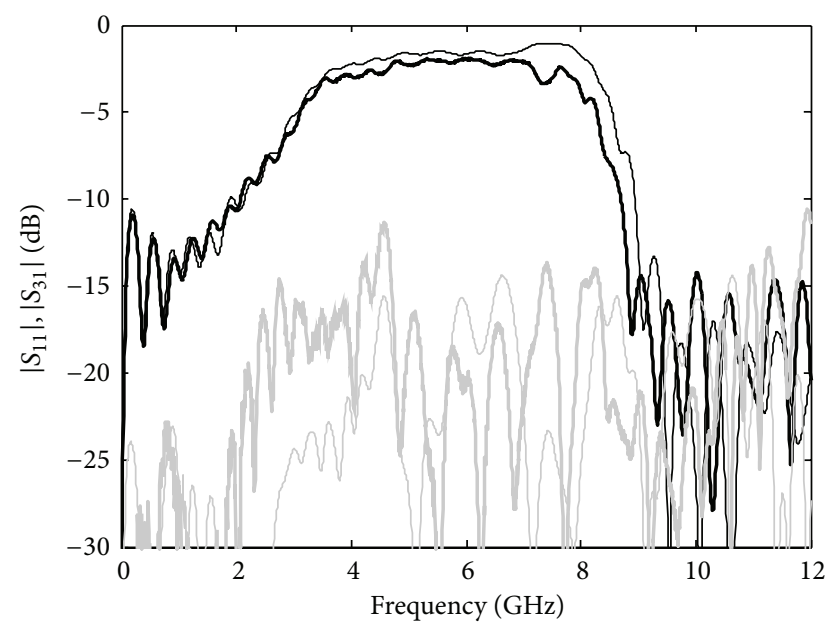

FIGURE 6: Simulations (thin line), and measurements (thick line) of $\left|S_{11}\right|$ (grey line) and $\left|S_{31}\right|$ (black line) in the dispersive delay line of Figure 5.

4.4. Compact Design of High-Power Spurious-Free LowPass Waveguide Filters for Satellite Payloads. High-power spurious-free low-pass rectangular waveguide filters are key components at the output of a satellite Output Multiplexer (OMUX) [26]. As stated in Section 3.2, if the amplitude of the sinusoidal coupling coefficient is high enough, each

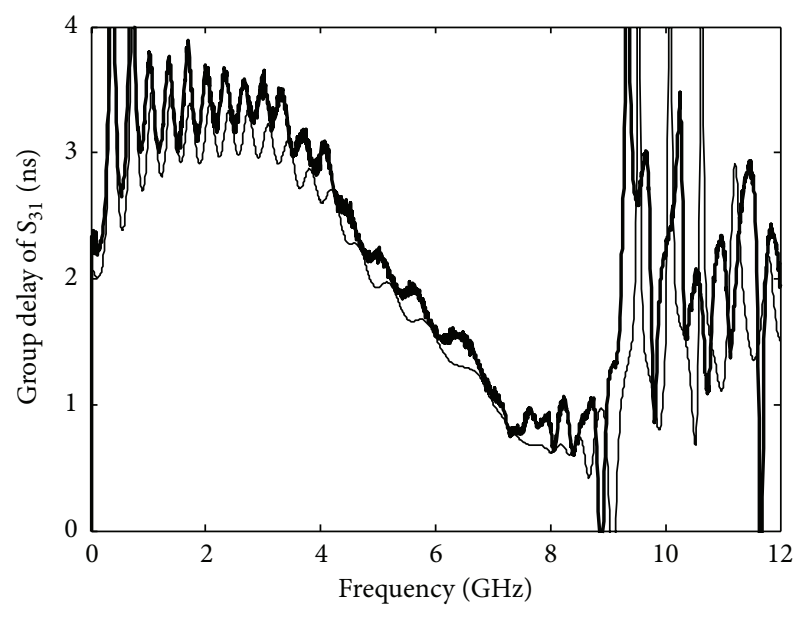

FIGURE 7: Simulation (thin line) and measurement (thick line) of the group delay of the $S_{31}$ parameter in the dispersive delay line of Figure 5 .

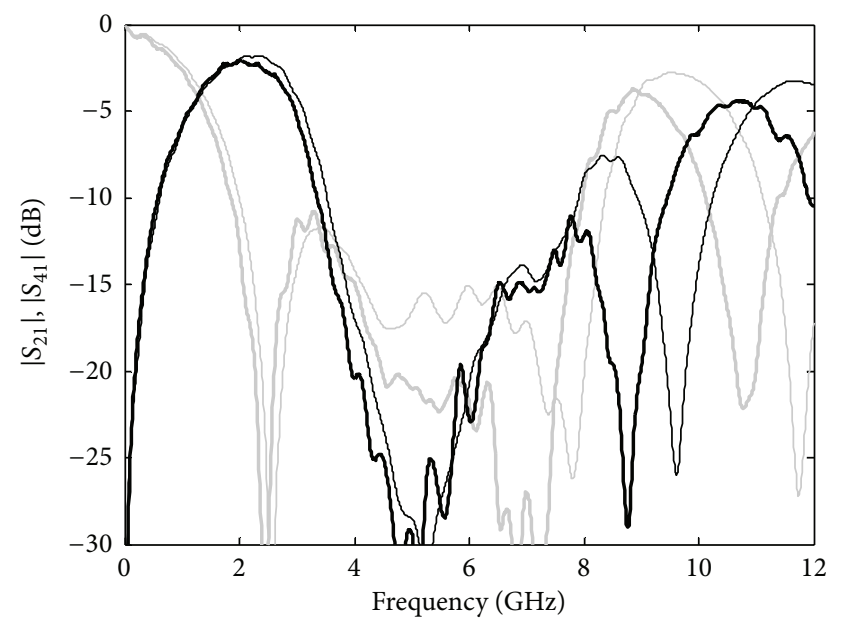

FIGURE 8: Simulations (thin line) and measurements (thick line) of $\left|S_{21}\right|$ (grey line) and $\left|S_{41}\right|$ (black line) in the dispersive delay line of Figure 5 .

corrugation behaves like an individual bandstop element whose height is around $\lambda_{g} / 4$, being $\lambda_{g}$ the guide wavelength of the fundamental $\mathrm{TE}_{10}$ mode at the frequency to be rejected [11], see Figure 9.

The resulting device for a passband between $10.7 \mathrm{GHz}$ and $12.7 \mathrm{GHz}$ with return losses around $20 \mathrm{~dB}$, and a $60 \mathrm{~dB}$ rejection level for frequencies over $13.75 \mathrm{GHz}$ and up to the third harmonic (around $40 \mathrm{GHz}$ ) has been manufactured in copper by electroforming. The photograph of the fabricated prototype is given in Figure 10(a), and in Figure 10(b) we show the simulations using CST Microwave Studio (grey line) and the measurements (black line) that have been performed by means of an Agilent 8722 Vector Network Analyzer, proper waveguide-to-coaxial transitions, and Maury $7005 \mathrm{E}$ calibration kits. 


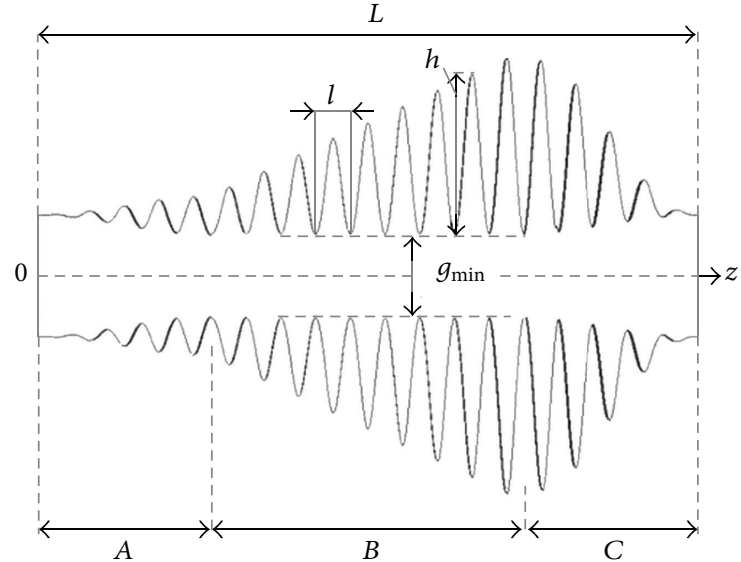

(a)

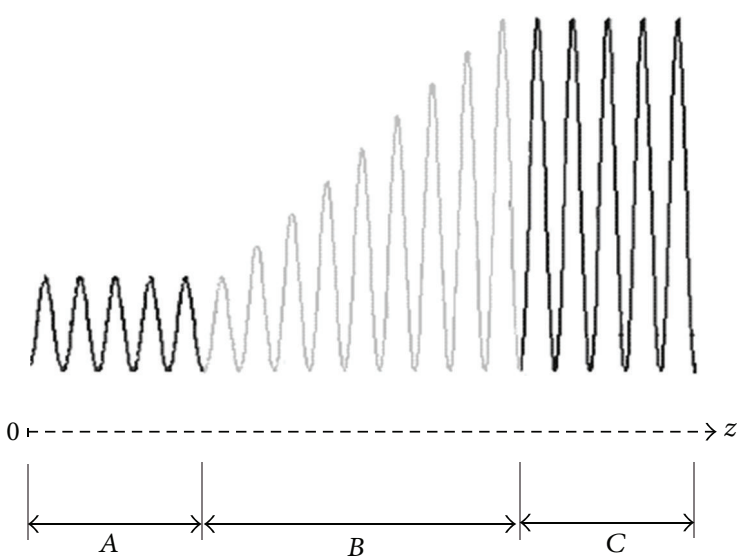

(c)

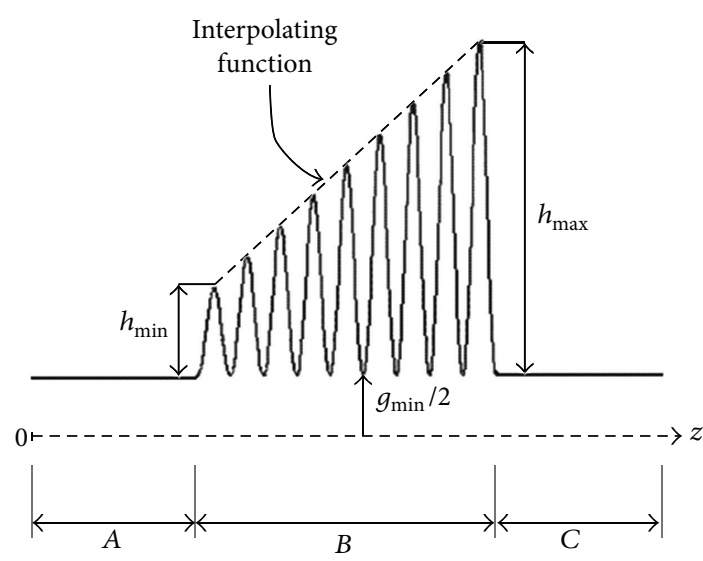

(b)

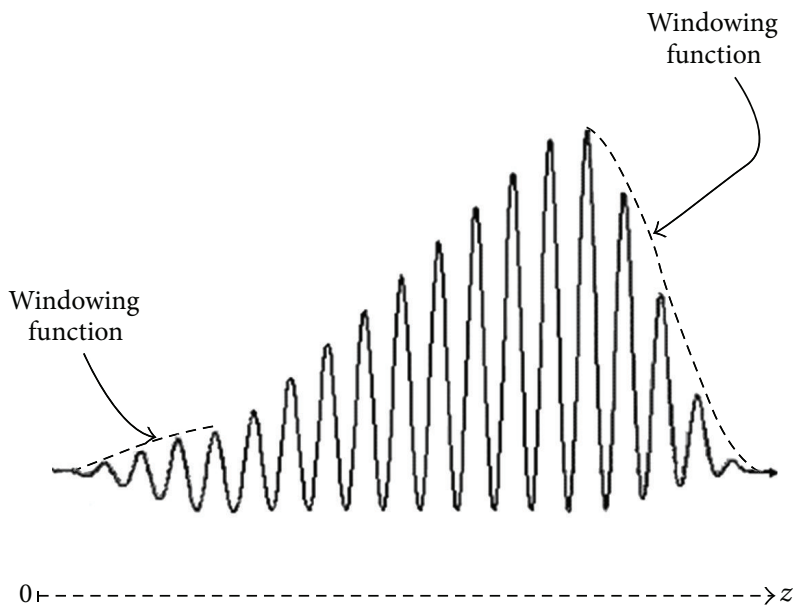

(d)

FIGURE 9: Schematics showing the design process of novel high-power spurious-free low-pass waveguide filters: (a) sketch showing the final filter profile and its physical parameters; (b) shaping of section $B\left(g_{\min }, h_{\min }\right.$ and $\left.h_{\max }\right)$; (c) new bandstop elements added to define sections $A$ and $C$; and (d) windowing applied to sections $A$ and $C$.

4.5. Pulse Shapers for Advanced UWB Radar and Communications and for Novel Breast Cancer Detection Systems. A new strategy for generating customized pulse shapes intended for use in wideband applications has been proposed and demonstrated [27]. The strategy employs the exact analytical series solution proposed in Section 3.3, [19]. Time-domain measurements are performed demonstrating the generation of two pulse shapes using microstrip technology. The first one satisfies the preestablished UWB mask requirements for advanced radar and ultrafast communication systems [28], see Figures 11, 12, and 13. The second one improves a timedomain microwave breast imaging system [29], see Figures 14 and 15.

4.6. Transmission-Type Nth-Order Differentiators for Tunable Pulse Generation. Nth-order differentiators are very promising devices for temporal analog processing and for the design of advanced tunable pulse generators [30]. Their general mathematical expression is $\widetilde{H}_{N}(f)=(2 \pi \cdot j \cdot f)^{N}$, where $j=$ $\sqrt{-1}$. Here, a 4 th-order differentiator has been designed for a frequency range from 3.1 to $7.4 \mathrm{GHz}$, which gives a good tradeoff between energy efficiency and maximum bandwidth for UWB signals, $H_{4}(f)$.

Following the synthesis procedure detailed in Section 3.3, the required coupling coefficient, $K(z)$, is calculated for the target frequency response, $H_{4}(f)$, using (10). The device will be implemented in coupled lines to work effectively in transmission and, therefore, the even and odd characteristic impedance, $Z_{0 e}(z)$ and $Z_{0 o}(z)$, need to be separated and windowed to keep the ports matched. Since microstrip technology will be used to implement the resulting device, the even and odd modes will propagate at different velocities, making the necessary to apply a compensation algorithm [30].

The resulting characteristic impedances together with the photograph of the fabricated prototype are shown in Figure 16. Figure 17 shows a very good agreement between the simulation using Agilent ADS Momentum (grey dotted line) and measurement results for the magnitude and phase of the $S_{31}(f)$ parameter (grey solid line) compared with 


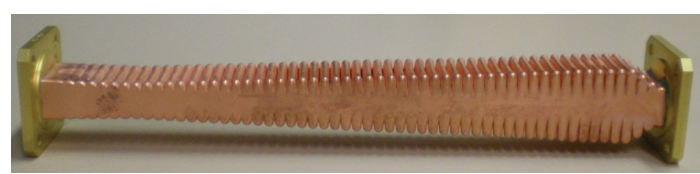

(a)

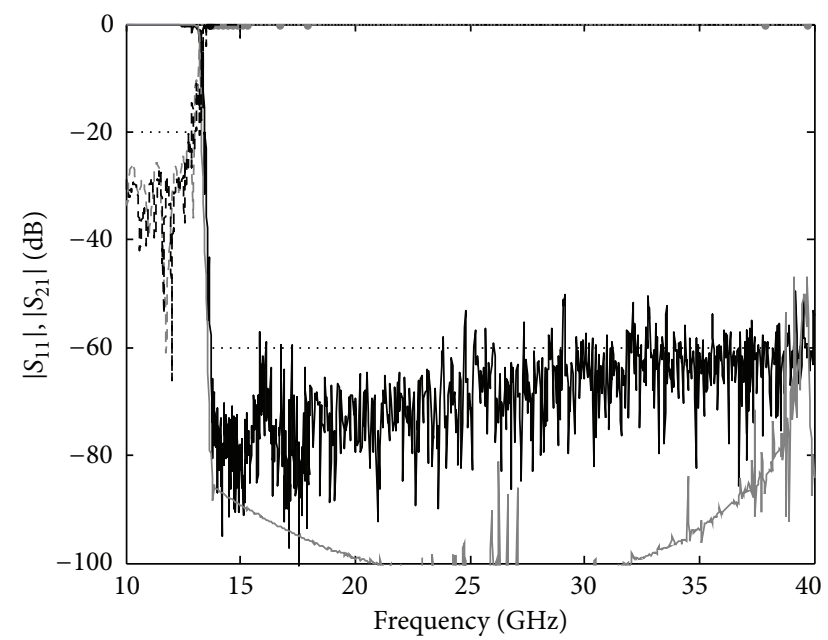

(b)

Figure 10: (a) Photograph of the fabricated prototype and (b) simulations (grey line) and measurements (black line) of the novel compact highpower spurious-free low-pass waveguide filter: $\left|S_{11}\right|$ in dashed line and $\left|S_{21}\right|$ in solid line. Frequency specifications for $\left|S_{11}\right|$ and $\left|S_{21}\right|$ are also given in dotted line.

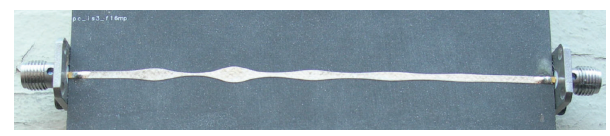

FIGURE 11: Photograph of a microstrip pulse shaper for UWB radar and communication systems.

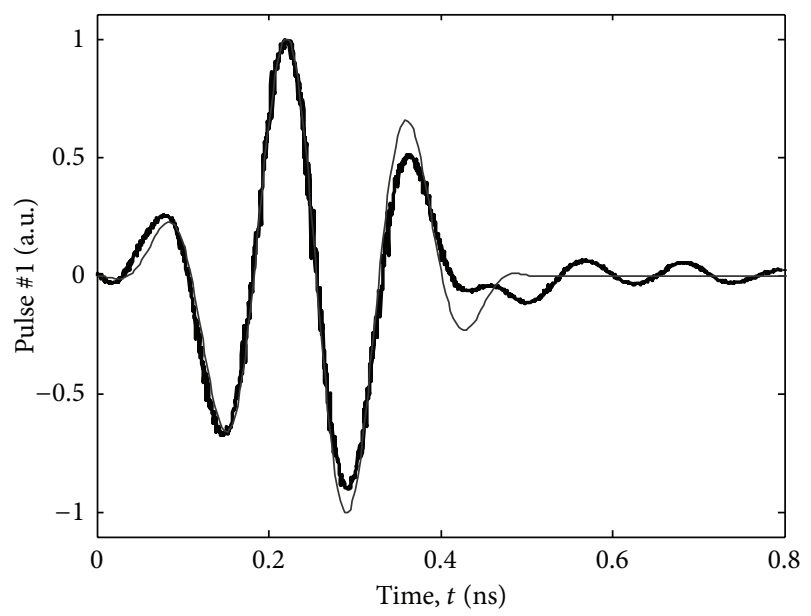

FIGURE 12: Performance of the pulse shaper for UWB radar and communications. Sinusoidal waveform windowed by a squared cosine: the target pulse (thin line), and the measurement (thick line).

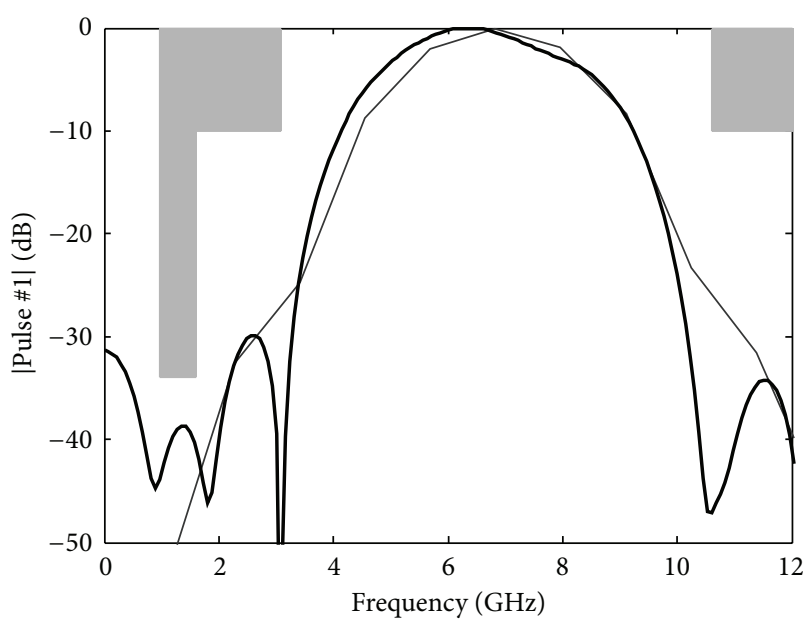

(a)

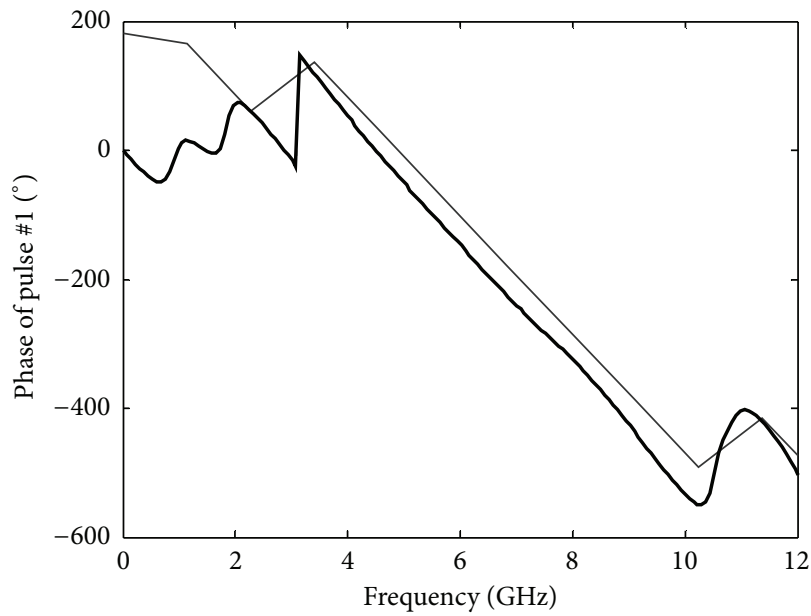

(b)

FIGURE 13: Spectrum (a) magnitude and (b) phase of the target signal (thin line) and Fourier-transformed measurement (thick line) of the pulse generated in the pulse shaper intended for UWB radar communications. The FCC UWB mask is also shown in grey.

the target specifications for the 4th-order differentiator (black dashed line). Moreover, the $\left|S_{11}\right|$ parameter (not shown) is below $-20 \mathrm{~dB}$ in the entire frequency range of interest, ensuring a good matching at the input port.

4.7. Robust Filter Design Tool: A Cauer Filter Example. The synthesis technique presented in Section 3.4 is incorporated in an exact robust filter design tool. The method is validated with an example in rectangular waveguide technology with WR-90 standard ports. The specifications are passbands from $8 \mathrm{GHz}$ to $11.4 \mathrm{GHz}$ and from $18.9 \mathrm{GHz}$ up to $22.5 \mathrm{GHz}$ with return losses better than $20 \mathrm{~dB}$ and a rejected band from $13 \mathrm{GHz}$ to $16.3 \mathrm{GHz}$ with a rejection level higher than $60 \mathrm{~dB}$. A bandpass frequency response following a Cauer (Elliptic) approximation [21,31] will be used for the $S_{11}$ parameter (a bandstop frequency response will result for the $S_{21}$ parameter). The order necessary for the approximation will be 14 , and 


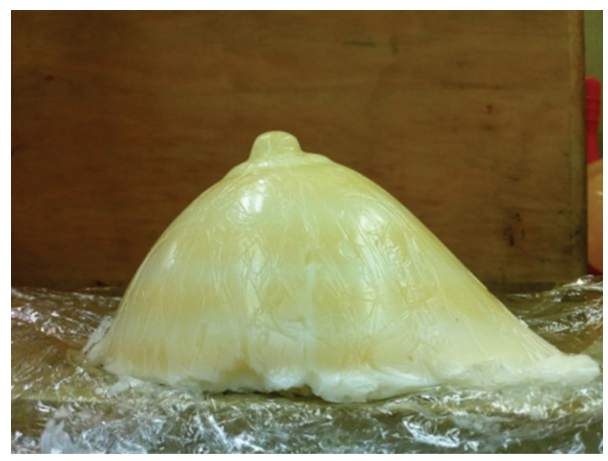

(a)

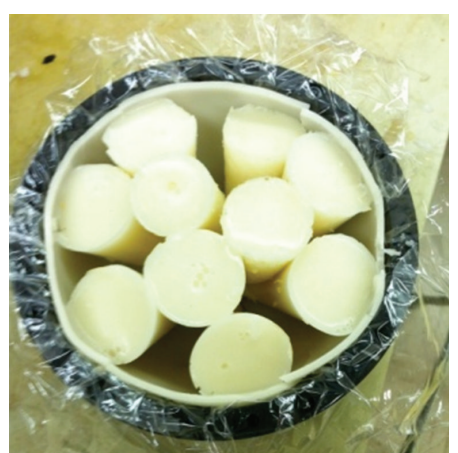

(b)

FIGURE 14: Two photographs of the heterogeneous breast phantoms: (a) exterior view of the realistically shaped breast phantom and (b) coronal view of the $2 \mathrm{~mm}$ skin layer and the conical gland structures embedded inside the phantom.

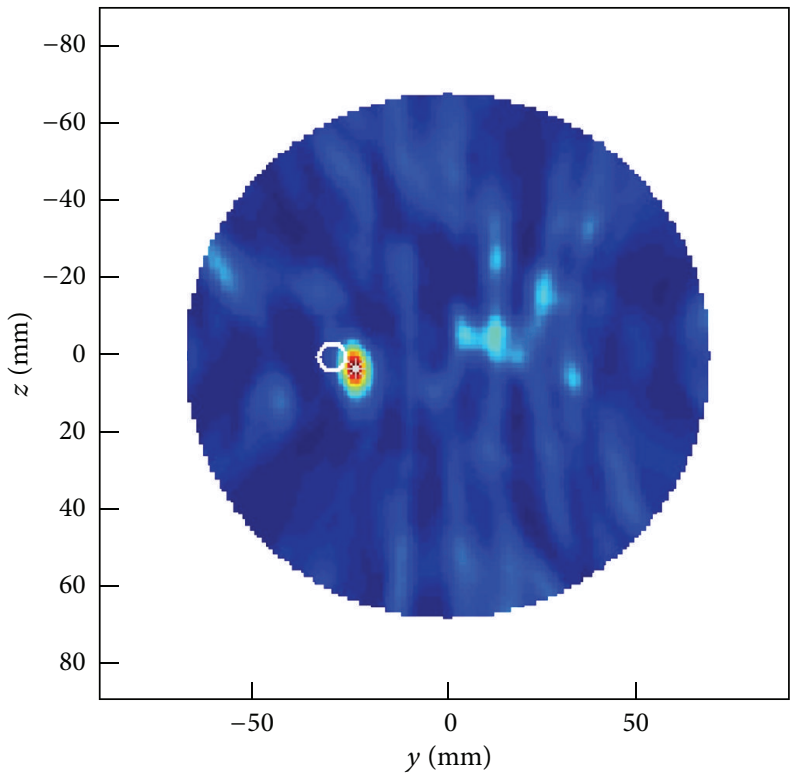

FIGURE 15: A coronal slice of the reconstructed 3D image of the realistically shaped adipose breast phantom using the designed pulse shaper. The dark red indicates higher intensity values corresponding to stronger EM scatterers. The "*” denotes the global maximum and the "o" represents the approximate tumour location based on the insertion of the tumour into the breast phantom.

the passband will extend from $13.1 \mathrm{GHz}$ up to $16.2 \mathrm{GHz}$, with a passband ripple of $10^{-9} \mathrm{~dB}$ and a rejected band ripple of $25 \mathrm{~dB}$. From the magnitude of the $S_{11}$ parameter, the magnitude of the $S_{21}$ can be immediately obtained by using (12), and a bandstop frequency response results for the $S_{21}$ parameter, with a rejected band attenuation of $67 \mathrm{~dB}$.

Following the expressions in Section 3.4, a prototype is designed and fabricated; see Figure 18. As it can be seen in Figure 19, the target, simulated using CST Microwave Studio, and measured frequency responses are in a very good agreement, satisfying the frequency requirements mentioned above, and confirming the accuracy and flexibility of the synthesis method proposed.

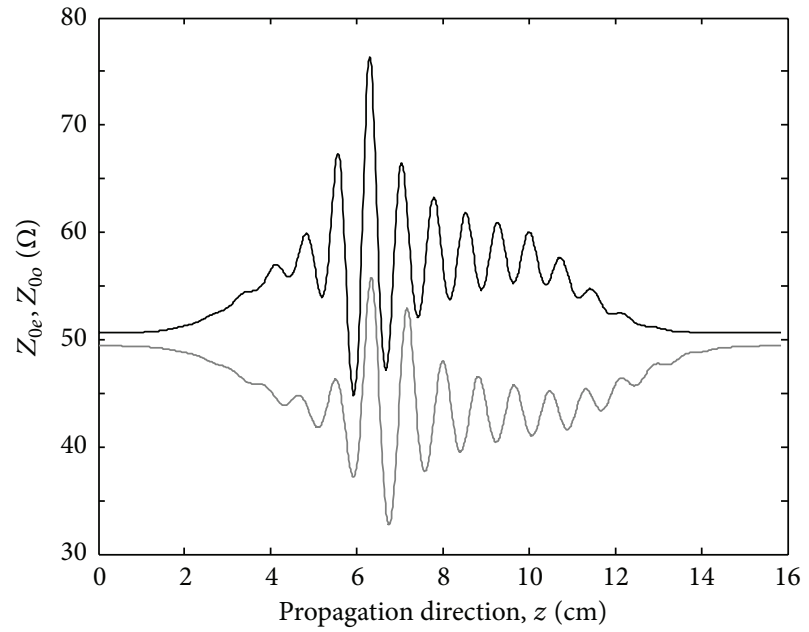

(a)

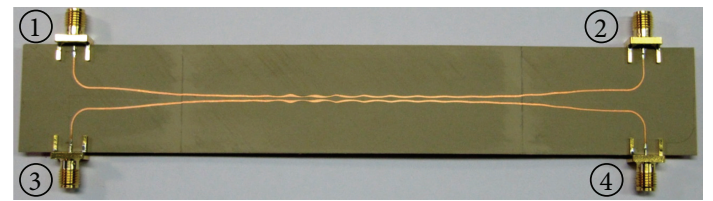

(b)

Figure 16: (a) Even (black line) and odd (grey line) characteristic impedance of the 4th-order differentiator after the compensation process and (b) the corresponding photograph of the prototype in microstrip technology for the given characteristic impedance.

\section{Conclusion}

New devices in microstrip technology, rectangular waveguide technology, and microstrip coupled line technology have been successfully designed, easily fabricated, and accurately tested for very different applications. A palate of Inverse Scattering microwave synthesis methods have been surveyed along the paper highlighting their advantages and disadvantages and confirming that all the techniques together represent a powerful tool for the design of passive microwave components for the new emerging applications in the wireless, radar, biomedical engineering, and satellite worlds. 


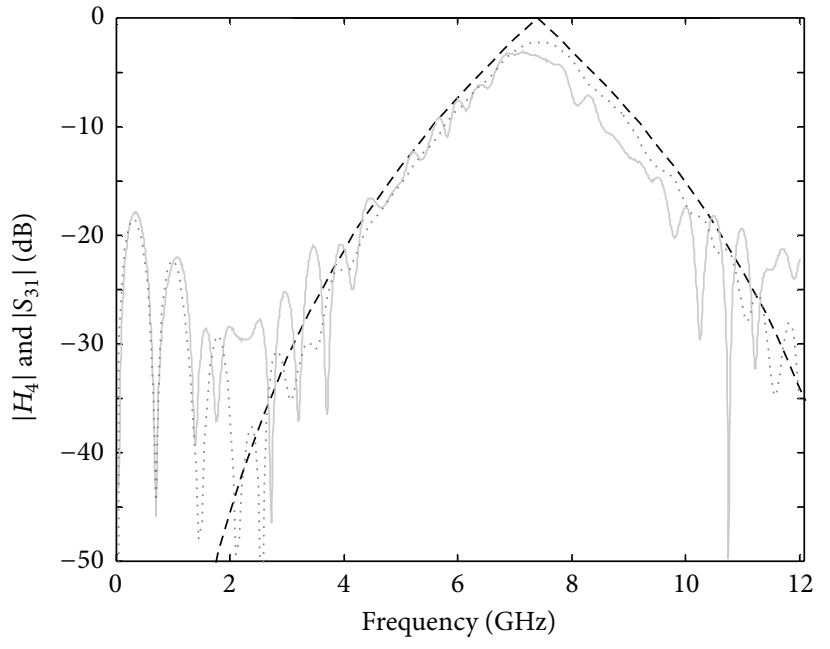

(a)

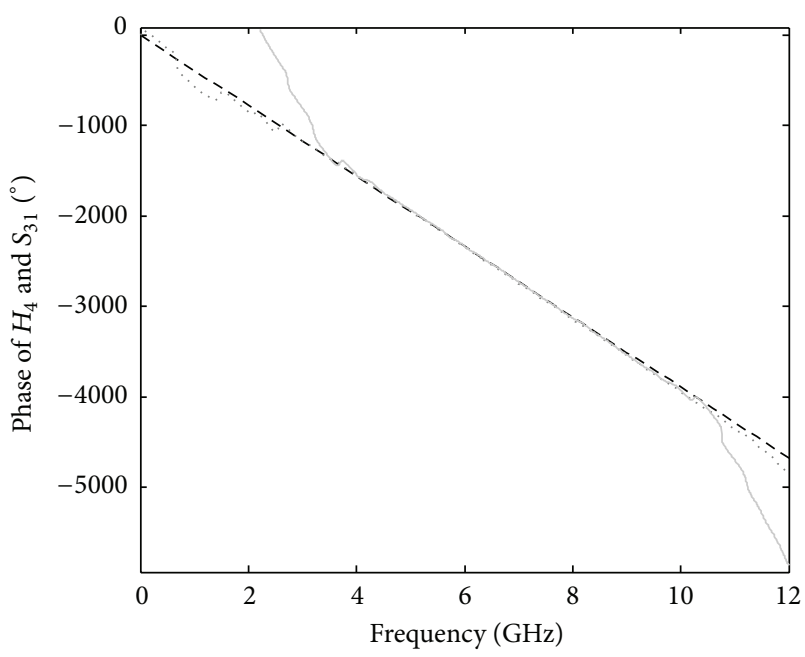

(b)

Figure 17: Target (black dashed line), simulated (dark grey dotted line), and measured (light grey solid line) magnitude (a) and phase (b) of the frequency response of the 4 th-order differentiator.

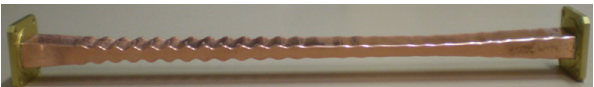

FIGURE 18: Photograph of the fabricated filter prototype in rectangular waveguide technology. Standard WR-90 ports are used.

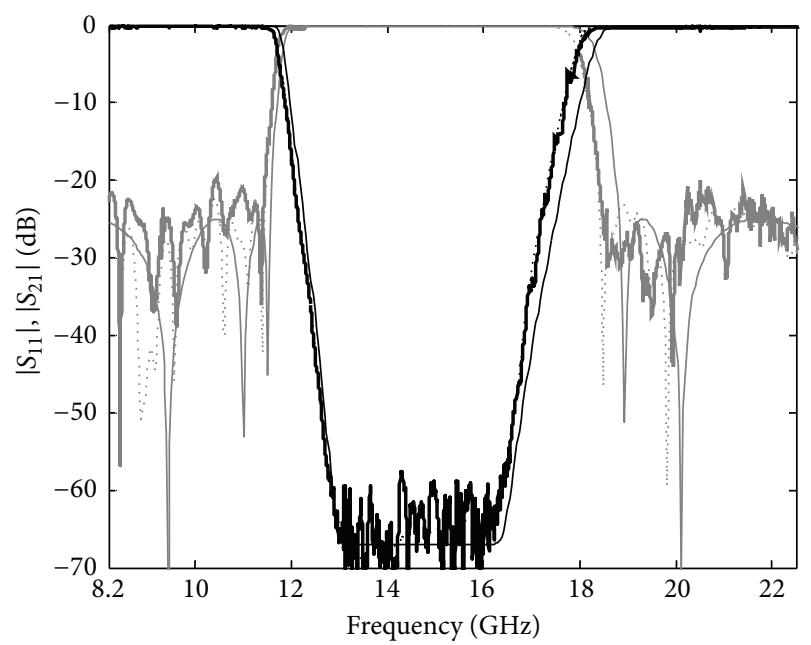

FIgURE 19: Magnitude of the $S_{11}$-parameter (grey line) and of the $S_{21}$ parameter (black line) for the final synthesized filter in waveguide technology in Figure 18. The target (thin solid line), simulated (dotted line), and measured (thick solid line) frequency responses are given.

\section{Acknowledgments}

This work has been supported by the Spanish Ministerio de Ciencia e Innovación through the Project TEC2011-28664C02-01. Magdalena Chudzik would like also to acknowledge Spanish Ministerio de Educación for its FPU Grant.

\section{References}

[1] D. C. Youla, "Analysis and synthesis of arbitrarily terminated lossless nonuniform lines," IEEE Transactions on Circuit Theory, vol. CT-11, pp. 363-372, 1964.

[2] R. W. Klopfenstein, "A transmission-line taper of improved design," Proceedings of the IRE, vol. 44, pp. 31-35, 1956.

[3] M. D. Abouzahra and L. Lewin, "Radiation from microstrip discontinuities," IEEE Transactions on Microwave Theory and Techniques, vol. MTT-27, no. 8, pp. 722-723, 1979.

[4] I. Arregui, I. Arnedo, T. Lopetegi, M. A. G. Laso, and A. Marcotegui, "Low-pass filter for electromagnetic signals," U. S. Patent 2010/0308938, European Patent 2244 330, January 21, 2008.

[5] C. Elachi, "Waves in active and passive periodic structures: a review," Proceedings of the IEEE, vol. 64, no. 12, pp. 1666-1698, 1976.

[6] Y. Qian, V. Radisic, and T. Itoh, "Simulation and experiment of photonic band-gap structures for microstrip circuits," in Proceedings of the 1997 Asia-Pacific Microwave Conference (APMC '97), pp. 585-588, Hong Kong, December 1997.

[7] F. Yang, R. Coccioll, Y. Qian, and T. Itoh, "Planar peg structures: basic properties and applications," IEICE Transactions on Electronics, vol. E83-C, no. 5, pp. 687-696, 2000.

[8] I. Arnedo, M. Chudzik, J. D. Schwartz et al., "Analytical solution for the design of planar electromagnetic bandgap structures with spurious-free frequency response," Microwave and Optical Technology Letters, vol. 54, no. 4, pp. 956-960, 2012.

[9] T. Lopetegi, M. A. G. Laso, R. Gonzalo et al., "Electromagnetic crystals in microstrip technology," Optical and Quantum Electronics, vol. 34, no. 1-3, pp. 279-295, 2002.

[10] M. A. G. Laso, T. Lopetegi, M. J. Erro et al., "Real-time spectrum analysis in microstrip technology," IEEE Transactions on Microwave Theory and Techniques, vol. 51, no. 3, pp. 705-717, 2003.

[11] I. Arregui, I. Arnedo, A. Lujambio et al., "A compact design of high-power spurious-free low-pass waveguide filter," IEEE 
Microwave and Wireless Components Letters, vol. 20, no. 11, pp. 595-597, 2010.

[12] E. F. Bolinder, "The relationship of physical applications of fourier transforms in various fields of wave theory and circuitry," IEEE Transactions on Microwave Theory and Techniques, pp. 153-158, 1957.

[13] B. Z. Katsenelenbaum, L. Mercader, M. Pereyaslavets, M. Sorolla, and M. Thumm, Theory of Nonuniform Waveguides: The Cross-Section Method, vol. 44 of IEE Electromagnetic Waves Series, IET, London, UK, 1998.

[14] F. Sporleder and H. G. Unger, Waveguide Tapers, Transitions and Couplers, Peter Peregrinus, London, UK, 1979.

[15] I. Arnedo, J. Gil, N. Ortiz et al., "Ku-band high-power lowpass filter with spurious rejection," Electronics Letters, vol. 42, no. 25, pp. 1460-1461, 2006.

[16] I. Arregui, I. Arnedo, A. Lujambio et al., "Design method for satellite output multiplexer low-pass filters exhibiting spuriousfree frequency behavior and high-power operation," Microwave and Optical Technology Letters, vol. 52, no. 8, pp. 1724-1728, 2010.

[17] J. D. Schwartz, I. Arnedo, M. A. G. Laso, T. Lopetegi, J. Azaña, and D. Plant, "An electronic UWB continuously tunable timedelay system with nanosecond delays," IEEE Microwave and Wireless Components Letters, vol. 18, no. 2, pp. 103-105, 2008.

[18] I. Arregui, F. Teberio, I. Arnedo et al., "High-power low-pass harmonic waveguide filter with TEn0-mode suppression," IEEE Microwave and Wireless Components Letters, vol. 22, no. 7, pp. 339-341, 2012.

[19] I. Arnedo, M. A. G. Laso, F. Falcone, D. Benito, and T. Lopetegi, "A series solution for the single-mode synthesis problem based on the coupled-mode theory," IEEE Transactions on Microwave Theory and Techniques, vol. 56, no. 2, pp. 457-466, 2008.

[20] M. Chudzik, I. Arnedo, I. Arregui et al., "Novel synthesis technique for microwave circuits based on inverse scattering: efficient algorithm implementation and application," International Journal of RF and Microwave Computer-Aided Engineering, vol. 21, no. 2, pp. 164-173, 2011.

[21] I. Arnedo, I. Arregui, A. Lujambio, M. Chudzik, M. A. G. Laso, and T. Lopetegi, "Synthesis of microwave filters by inverse scattering using a closed-form expression valid for rational frequency responses," IEEE Transactions on Microwave Theory and Techniques, vol. 60, no. 5, pp. 1244-1257, 2012.

[22] M. J. Erro, I. Arnedo, M. A. G. Laso, T. Lopetegi, and M. A. Muriel, "Phase-reconstruction in photonic crystals from Sparameter magnitude in microstrip technology," Optical and Quantum Electronics, vol. 39, no. 4-6, pp. 321-331, 2007.

[23] M. Chudzik, I. Arnedo, A. Lujambio et al., "Microstrip coupledline directional coupler with enhanced coupling based on EBG concept," Electronics Letters, vol. 47, no. 23, pp. 1284-1286, 2011.

[24] C. Caloz, "Metamaterial dispersion engineering concepts and applications," Proceedings of the IEEE, vol. 99, no. 10, pp. 17111719, 2011.

[25] A. Lujambio, I. Arnedo, M. Chudzik, I. Arregui, T. Lopetegi, and M. A. G. Laso, "Dispersive delay line with effective transmission-type operation in coupled-line technology," IEEE Microwave and Wireless Components Letters, vol. 21, no. 9, pp. 459-461, 2011.

[26] P. Fortescue and J. Stark, Spacecraft Systems Engineering, Wiley, Chichester, UK, 1995.

[27] Part 15 Rules for Unlicensed RF Devices, Federal Communications Commission (FCC), September 2007, http://www.fcc.gov/ oet/info/rules.
[28] I. Amedo, J. D. Schwartz, M. A. G. Laso, T. Lopetegi, D. V. Plant, and J. Azaña, "Passive microwave planar circuits for arbitrary UWB pulse shaping," IEEE Microwave and Wireless Components Letters, vol. 18, no. 7, pp. 452-454, 2008.

[29] A. Santorelli, M. Chudzik, E. Kirshin et al., "Experimental demonstration of pulse shaping for time-domain microwave brest imaging," Progress in Electromagnetics Research, vol. 133, pp. 309-329, 2013.

[30] M. Chudzik, I. Arnedo, A. Lujambio et al., "Design of transmission-type Nth-order differentiators in planar microwave technology," IEEE Transactions on Microwave Theory and Techniques, vol. 60, no. 11, pp. 3384-3394, 2012.

[31] I. Arnedo, A. Lujambio, T. Lopetegi, and M. A. G. Laso, "Design of microwave filters with arbitrary frequency response based on digital methods," IEEE Microwave and Wireless Components Letters, vol. 17, no. 9, pp. 634-636, 2007. 

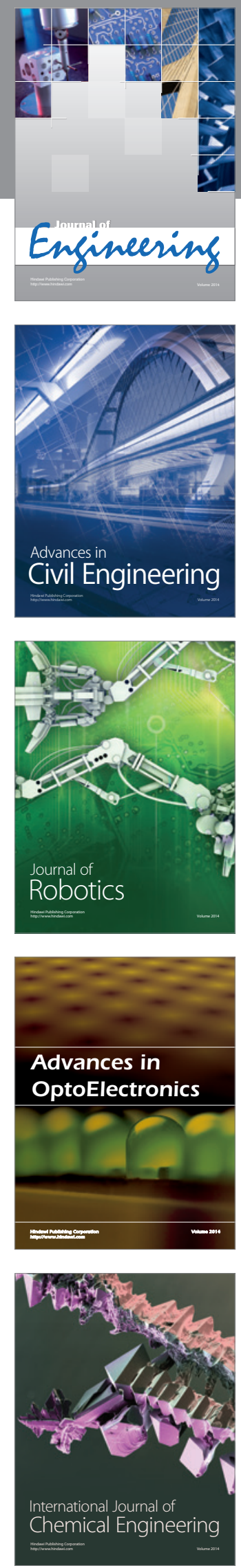

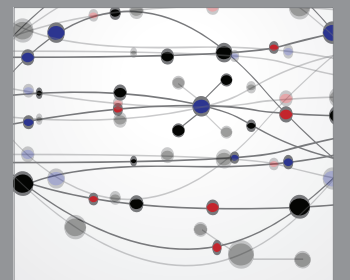

The Scientific World Journal
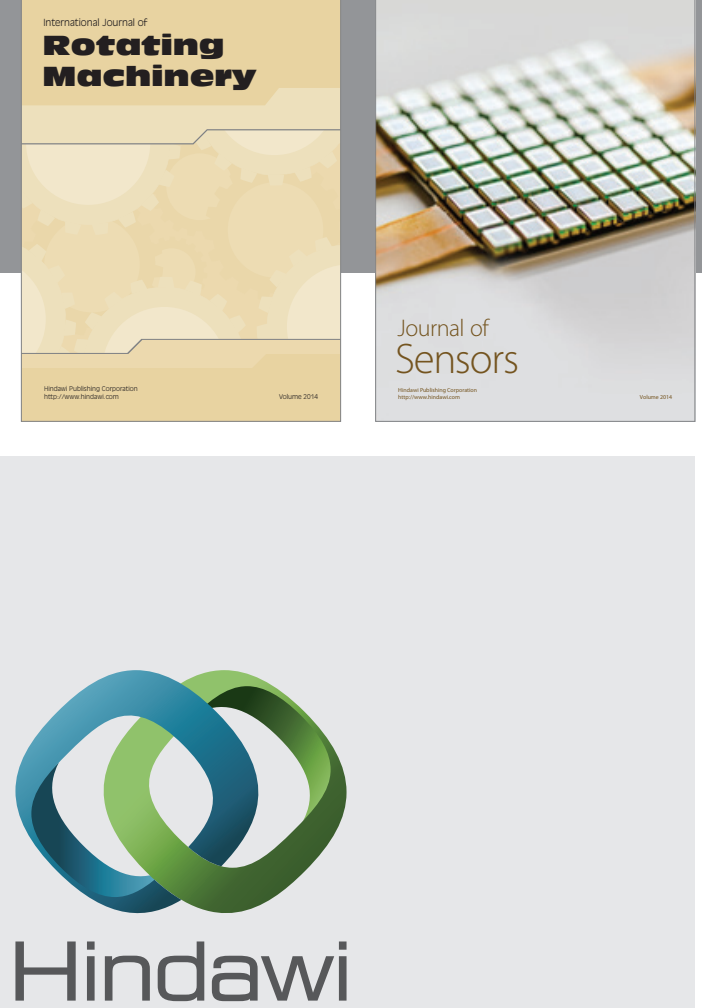

Submit your manuscripts at http://www.hindawi.com
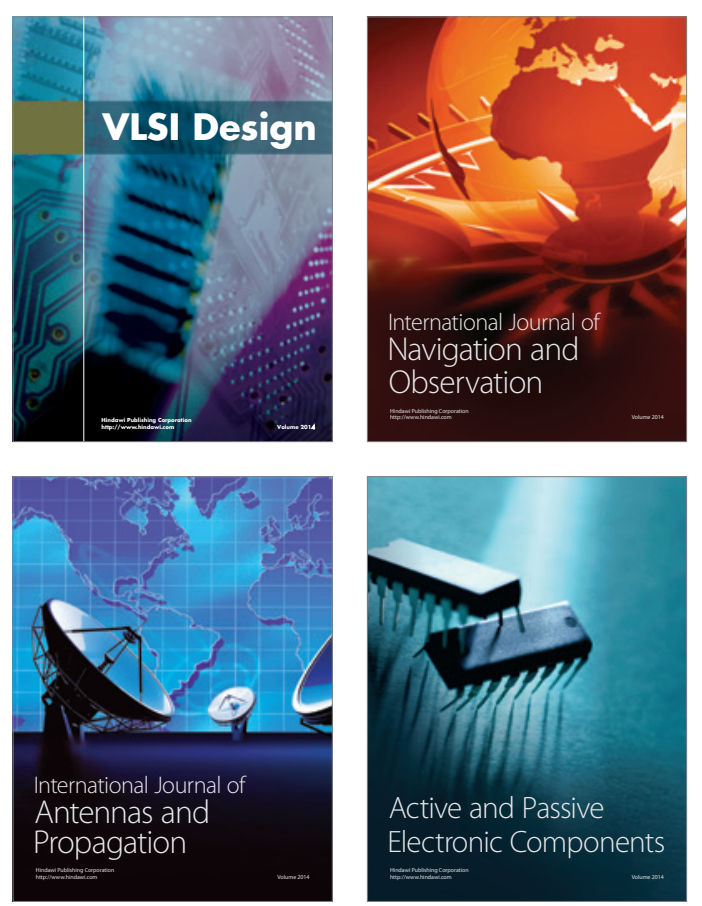
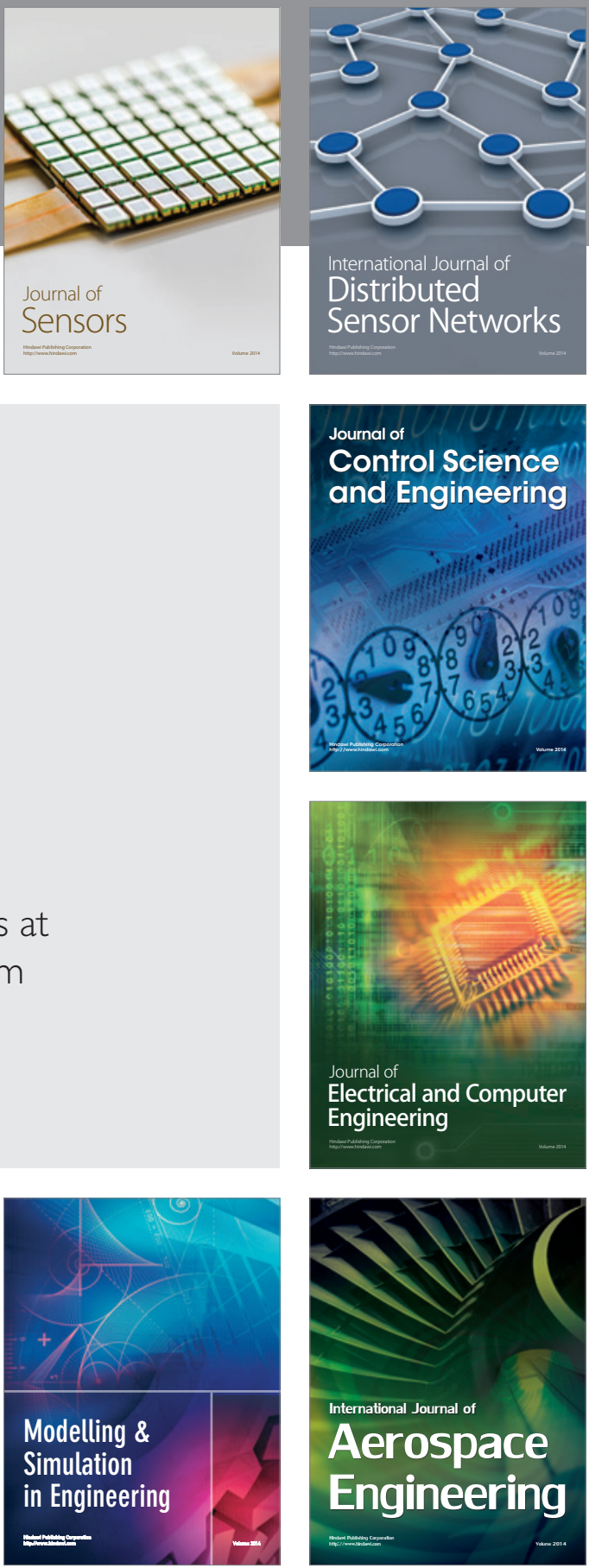

Journal of

Control Science

and Engineering
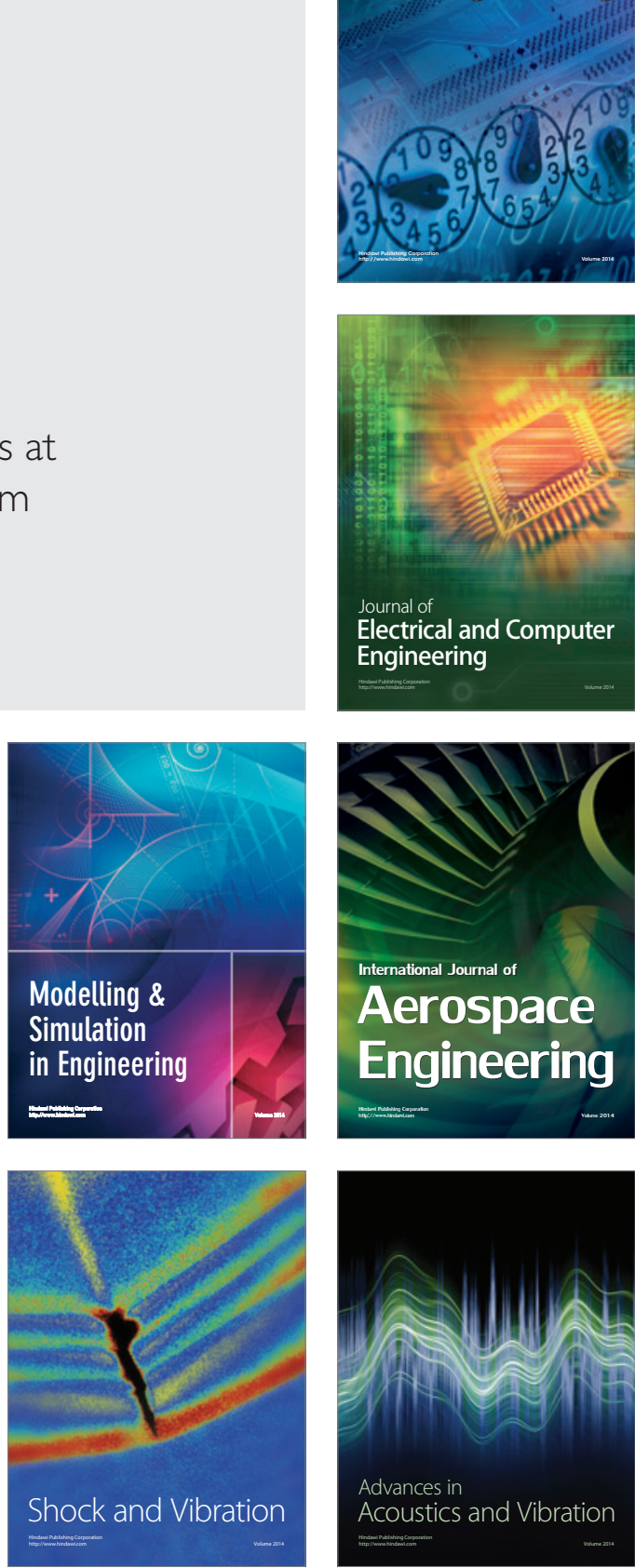